\title{
A Novel Transmit Optimization Scheme for MIMO Spatial Multiplexing Systems
}

\author{
Yan Zhu, JinJing Jiang, Cong Shen and Jing Wang \\ Department of Electronic Engineering \\ Tsinghua University \\ Beijing, China 100084 \\ Telephone: (8610) 6278-1398 \\ Fax: (8610) 6277-0317 \\ Email: mite98@mails.tsinghua.edu.cn
}

\begin{abstract}
In this paper, we propose a new kind of adaptive adaptive MIMO signaling scheme . Compared with other adaptive scheme, our approach utilize both the channel state information (CSI) and the constellation of the information streams. We also find that some precoders can be greatly enhanced by the constellation rotation, while others achieve nearly no improvement. We analyze this phenomenon and therefore develop some criteria to generalize the combination of them. Numerical simulations are presented and verify our results.
\end{abstract}

\section{INTRODUCTION}

Recently, multi-antenna system has been widely studied due to its attractive advantages, such as high transmit rate and high reliability. Open-loop system, i.e. only receiver knows the channel state information have been studied firstly, such as V-BLAST [1], Almouti Code, Linear Dispersion (LD) Code and so on. Recently, the adaptive space-time processing is becoming a new highlight motivated by the need for higher transmission rate and better performance. Many techniques have made it possible for the transmitter to obtain the full or partial channel knowledge. For example, channel state information (CSI) can be fed back to the transmitter through some physical links provided the channel does not change quickly, i.e. it can be viewed as unchanged during a certain period. In such scenarios, the joint optimization of transmit signaling and receive detection can greatly improve the overall performance. [5] and [6] have demonstrated that even the linear processing will bring significant performance improvement to the whole system. In [5], the authors unify a class of precoders by using the general weighed-MMSE precoder. Some new types of precoders are developed based on this unification, such as equal error precoder. In [6], the authors also develop some optimal precoders which targeted minimum MSE and BER, under constraints on the transmit power or the maximum output of the transmitter.

Constellation rotation is a scheme for open-loop systems. It was first introduced in the context of single-in-singleout (SISO) system [2], in which the authors analyze the signal space diversity. Xin has used it for the space-time diversity system [3] and developed a new class of space-time block code (STBC), which can achieve high performance in quasi-static channel. Using rotation technique, we can get not only the full diversity gain but also high coding gain on a group of parallel channels [4]. It is a promising technique in the research of MIMO.

However, both of the constellation rotation and the precoder scheme only consider partial factor in the whole communication system: Linear precoder only uses the CSI to get optimal processing and it does not use the information about constellation; while constellation rotation only extracts the characteristics of the constellation without utilizing the channel information. So better performance can be expected if we design the transmit and receive scheme based on both the CSI and the characteristics of the constellation. In this paper, we will combine these two techniques together and develop some criteria to optimize the system. Our scheme efficiently utilize both the CSI and the constellation information and great performance improvement is achieved in some situations. However, we also find that not all the precoders can be enhanced by the constellation rotation. We analyze the problem and give the condition when the combination is efficient.

We organize the remaining parts of this paper as follows: In section II, the system model is described. The techniques of precoding and constellation rotation will be summarized in section III. Then we will discuss some results of the combination in the following section. Several numerical simulation results will be shown in section V. Finally, we come to the conclusion.

\section{System And Channel Model}

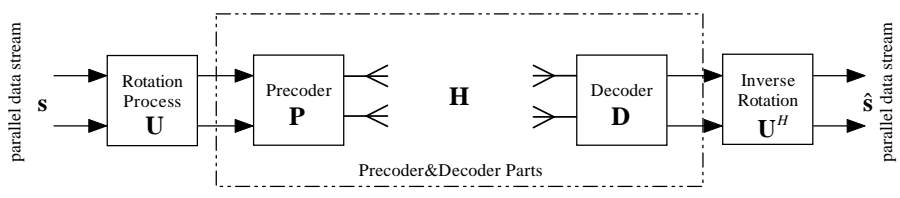

Fig. 1. MIMO communication system model 
We consider the wireless link with $n_{T}$ transmit and $n_{R}$ receive antennas, as shown in Fig. 1 . Let $h_{i j}$ be the complex path gain from transmit antenna $j$ to receive antenna $i$. We assume that $\left\{h_{i j}\right\}$ are independently complex normal distributed with unit variance $(\mathcal{C N}(0,1))$ and write $\mathbf{H}=\left[h_{i j}\right] \in \mathcal{C}$. We also assume that the channel matrix $\mathbf{H}$ remains invariant within a symbol period (with respect to each transmit antenna) and is known both at the receiver and the transmitter.

There are $q$ (usually, $q \leq n_{T}$ ) parallel symbol streams ( $\mathbf{s}=$ $\left[s_{1}, s_{2}, \ldots, s_{q}\right]^{\mathrm{T}}$ ) in total. Before going to the linear precoder $\mathbf{P}, \mathbf{s}$ has to be rotated by a unitary matrix $\mathbf{U}$. After being precoded by $\mathbf{P}$, the signal is sent into the channel. In order to recover the original $\mathbf{s}$, the receiver signal must be decoded by $\mathbf{D}$ and take an inverse rotation $\mathbf{U}^{\mathrm{H}}$. Under these assumptions, we have following express:

$$
\hat{\mathbf{s}}=\mathbf{U}^{\mathrm{H}} \mathbf{D}(\mathbf{H P U} \mathbf{s}+\mathbf{n})
$$

where ()$^{\mathrm{H}}$ represents the Hermitian transpose. $\mathbf{n}$ denotes the ambient noise $\left(E\left(\mathbf{n}^{\mathrm{H}} \mathbf{n}\right)=\mathbf{R}_{n n}\right)$. Because the whole system is power limited. We have the following constraints:

$$
\begin{aligned}
E\left(\mathbf{s}^{\mathrm{H}} \mathbf{s}\right) & =\sigma_{s}^{2} \mathbf{I} \\
\operatorname{tr}\left(\mathbf{U}^{\mathrm{H}} \mathbf{U}\right) & =q \\
\operatorname{tr}\left(\mathbf{P}^{\mathrm{H}} \mathbf{P}\right) & =p_{0}
\end{aligned}
$$

where $\operatorname{tr}()$ denotes the trace of the a matrix and $\sigma_{s}^{2}$ is the variance of each symbol stream. Thus the total transmit power is less than $\sigma_{s}^{2} p_{0}$. It is easy to show that $\mathbf{s}, \hat{\mathbf{s}}$ are both $q \times 1$ and that $\mathbf{P}, \mathbf{D}$ and $\mathbf{U}$ are matrices $n_{T} \times q, n_{R} \times q$ and $q \times q$ respectively.

\section{PRECODER AND CONSTEllation Rotation REVISITED}

In this section, we will briefly introduce the concepts of the linear precoder and constellation rotation. We will also demonstrate the natural relationship between the precoding and the constellation rotation.

\section{A. Linear Precoder and Decoder}

The system model of a normal linear precoder is the part of Fig. 1 without the rotation matrix $\mathbf{U}$, i.e. the symbol streams go into the precoder $\mathbf{P}$ directly and do not need the inverse rotation at the receiver, either. The optimal linear precoder design is to find the optimal precoder $\mathbf{P}$ and decoder $\mathbf{D}$, using the CSI, i.e. H. It can be summarized by the following optimization problem:

$$
\min _{\mathbf{P}, \mathbf{D}} L(\mathbf{P}, \mathbf{D})=E\left[\mathbf{e}^{\mathrm{H}} \mathbf{W}^{\mathrm{H} / 2} \mathbf{W}^{1 / 2} \mathbf{e}\right]
$$

subject to

$$
\operatorname{tr}\left(\mathbf{P}^{\mathrm{H}} \mathbf{P}\right) \leq p_{0}
$$

Function $L(\mathbf{P}, \mathbf{D})$ is a cost function or so-called objective function. (6) is the power constraint. $\mathbf{e} \triangleq \mathbf{s}-(\mathbf{D H P s}+\mathbf{D n})$ and $\mathbf{W}$ is the $q \times q$ positive definite weight matrix. Various Ws can be used to derive various optimal precoders. There is a general form of the solution to the above problem: [7]

$$
\begin{aligned}
\mathbf{P} & =\mathbf{V} \boldsymbol{\phi}_{\boldsymbol{p}} \\
\mathbf{D} & =\boldsymbol{\phi}_{\boldsymbol{d}} \mathbf{V}^{\mathrm{H}} \mathbf{H}^{\mathrm{H}} \mathbf{R}_{n n}^{-1}
\end{aligned}
$$

where $\mathbf{R}_{n n}=E\left[\mathbf{n} \mathbf{n}^{\mathrm{H}}\right]$ and $\mathbf{V}$ is determined by eigen value decomposition of $\mathbf{H}^{\mathrm{H}} \mathbf{R}_{n n}^{-1} \mathbf{H}$ as following:

$$
\mathbf{H}^{\mathrm{H}} \mathbf{R}_{n n}^{-1} \mathbf{H}=\left[\begin{array}{ll}
\mathbf{V} & \tilde{\mathbf{V}}
\end{array}\right]\left[\begin{array}{cc}
\Lambda & 0 \\
0 & \tilde{\Lambda}
\end{array}\right]\left[\begin{array}{ll}
\mathbf{V} & \tilde{\mathbf{V}}
\end{array}\right]^{\mathrm{H}}
$$

$\Lambda$ is a diagonal matrix with the first largest $q$ eigen values of $\mathbf{H}^{\mathrm{H}} \mathbf{R}_{n n}^{-1} \mathbf{H}$ on its diagonal. $\mathbf{V}$ is composed of all the eigen vectors corresponding to $\Lambda$.

$\boldsymbol{\phi}_{p}$ and $\boldsymbol{\phi}_{\boldsymbol{d}}$ are both diagonal matrices. Usually, if $\mathbf{W}$ is fixed $\boldsymbol{\phi}_{p}$ will be determined completely, while $\boldsymbol{\phi}_{d}$ is determined by detection algorithm, such as zero-force (ZF). Assigning various expresses to $\mathbf{W}$, we can get a series of optimal precoders, such as maximum information rate design, un-weighted MMSE design, equal error design and so on. Because transmission has been optimized, the performance should be better than the one that CSI is only known to the receiver.

It is easy to find all of these kinds of designs make DHP diagonalized. That is to say, the effect of $\mathbf{D}$ and $\mathbf{P}$ make the original "mixture" channel $\mathbf{H}$ become a group of independent parallel channels(see Fig. 2) This equivalent channel model can be used by the constellation rotation technique [7].

\section{B. Constellation Rotation}

The main idea of constellation rotation is to introduce signal space diversity across the parallel symbol streams. Let $\mathbf{s}_{1}=\left[s_{11}, s_{12}, \ldots, s_{1 n_{T}}\right]$ be one valid code in the codebook, where $\left\{s_{1 i}\right\}_{i=1}^{n_{T}}$ are certain values in the constellation. Suppose that $\mathbf{s}_{2}$ is another valid code and we let $s_{21} \neq s_{11}$, while $s_{1 i}=s_{2 i}$ for $i=2 \ldots n_{T}$. Then $\mathbf{s}_{1}-\mathbf{s}_{2}=\left[s_{11}-s_{21}, 0, \ldots, 0\right]$. According to [4], this kind of code's diversity gain is only 1. To avoid this problem, we can introduce a unitary matrix $\mathbf{U}$, which can guarantee that for any two valid codes $\mathbf{s}_{1}$ and $\mathbf{s}_{2}, x_{i} \neq 0 \forall i$, where $\left[x_{1}, x_{2}, \ldots, x_{n_{T}}\right]^{\mathrm{T}}=\mathbf{U}\left(\mathbf{s}_{1}-\mathbf{s}_{2}\right)$. Therefore, after rotating the constellation, the diversity gain can be $n_{T}$. The system's asymptotic performance may be improved.

In [4], the author reduced the set of unitary matrices to the 
following form:

$$
\mathbf{U}=\frac{1}{\beta}\left[\begin{array}{cccc}
1 & \omega_{1} & \cdots & \omega_{1}^{q-1} \\
1 & \omega_{2} & \cdots & \omega_{2}^{q-1} \\
\vdots & \vdots & & \vdots \\
1 & \omega_{q} & \cdots & \omega_{q}^{q-1}
\end{array}\right]
$$

where $\beta$ is use to guarantee $\operatorname{tr}\left[\mathbf{U}^{\mathrm{H}} \mathbf{U}\right]=q .\left\{\omega_{i}\right\}_{i=1}^{q}$ are parameters needed to be optimized. The principle of design of $\left\{\omega_{i}\right\}_{i=1}^{q}$ is first to achieve the full diversity $n_{T}$ and then to achieve the coding gain as high as possible. This kind of optimization is determined by the modulation of the streams [4].

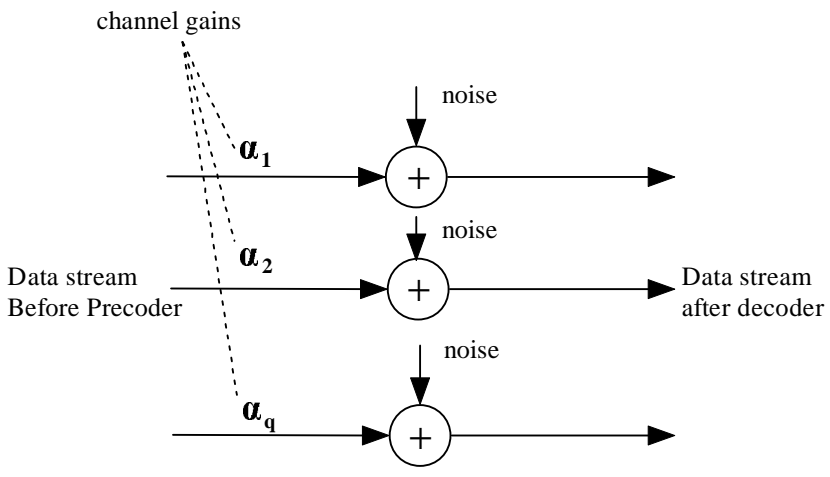

Fig. 2. Equivalent Channel Model with Precoder and Decoder

\section{NOVEL SCHEME WITH ROTATION AND DESIGN CRITERA}

In this section we will propose our new scheme and discuss some details about the generalization of the two techniques. These details will give us some hints to optimize the whole system.

As we have seen in section III, the procoder and decoder provide parallel channels for the constellation rotation. In this section, we have a formal conclusion as follows without strict proof:

Conclusion 1: The Precoder design and rotation design can be done separatively without any negative effects on each other, as long as the precoder matrix $\mathbf{P}$ and the decoder matrix $\mathbf{D}$ make $\mathbf{D H P}$ to be a diagonal matrix.

Remark: The design of precoders mainly depends on the channel information $(\mathbf{H})$ and has no constraint on the symbol stream except that $E\left[\mathbf{s s}^{\mathrm{H}}\right]=\sigma_{s}^{2} \mathbf{I}$. Because we limit our rotation matrix in the set of unitary ones, let $\tilde{\mathbf{s}}=\mathbf{U s}$, then $E\left[\tilde{\mathbf{s}} \tilde{\mathbf{s}}^{\mathrm{H}}\right]=E\left[\mathbf{U} \mathbf{s s}^{\mathrm{H}} \mathbf{U}^{\mathrm{H}}\right]=\sigma_{s}^{2} \mathbf{I}$. Thus the only constraint to the symbol streams is still hold after rotating. On the other hand, U only focuses on the parallel data streams' constellation without any more assumption about the channels expect that it must be parallel. As long as DHP is a diagonal matrix, the condition for using rotation is guaranteed. Therefore, Conclusion 1 is hold.
The above conclusion only tells us that the combination will not degrade the performance, but it does not guarantee the performance improvement of the whole system. There are really some precoders that can not be improved by constellation rotation. Now we give a sufficient condition for this phenomenon (maybe also a necessary one).

Conclusion 2: When DHP is a diagonal matrix, if $\left|\phi_{p, i}\right|^{2} \sigma_{s}^{2} \lambda_{i, i} \quad i=1 \ldots q$ is independent on $i$, there will be little improvement after rotating,s where $\phi_{p, i}$ and $\lambda_{i, i}$ are the $i$ th element on the diagonals of $\phi_{p}$ and $\Lambda$ respectively.

Remark: $\left|\phi_{p, i}\right|^{2} \sigma_{s}^{2} \lambda_{i, i}$ represents the $i$ th sub-channel's SNR [6]. If the value does not depend on $i$, that means all the sub-channels have the same SNR at any moment. In this scenario, the overall performance only depends on the Euclidean metrics among the valid codes. Because $\mathbf{U}$ is a unitary matrix, its action on the codebook does not change the original metric between any two codes. Thus, there is no improvement.

We do find some optimal precoders, which have such property. For example, the equal error design in [5] and maximum minima SNR in [6] are all this kind of precoders. Although the equivalent sub-channel SNR makes these precoders stronger than others, such as the un-weighted MMSE precoder [5], this property also prevents them to be improved further. The simulation in the following section will verify this point of view.

\section{NumericAl Simulation Results}

We give some results of our numerical simulation. In all the simulations, we assume that total transmit power across all transmit is normalized to unity $\left(p_{0}=1, \sigma_{s}^{2}=1\right)$. The SNR is defined as the ratio of total transmitting power to the noise variance at each receive antenna $\left(\mathbf{R}_{n n}=\sigma_{n}^{2} \mathbf{I}\right)$. $n_{T}$ and $n_{R}$ are both 5 and each symbol stream is uncoded QAM symbol. We use Monte Carlo simulations and in each experiment, we have run at least $10^{6}$ realizations of $\mathbf{H}$. For each type of precoder, we let $q=4$ and $q=5$ respectively. For $q=4$ we take $\omega_{i}=e^{j \pi(i-3 / 4)}$ to get the rotation matrix by (10) and for $q=5$ we take $\omega_{i}=\sqrt[5]{2} e^{j \pi(8 i-7) / 20}$. Both $\omega_{i}$ s are optimal for their own situations.

\section{A. Combinations with little improvement}

In this section, we present two precoders, equal error design and minimum PEP design, which have little improvement after rotating. Both of them make their sub-channel's SNRs constant. Fig. 3 and Fig. 4 show the BER performance under different SNRs. We can find there is little improvement with cooperating with the rotation. These results exactly verify Conclusion 2. 


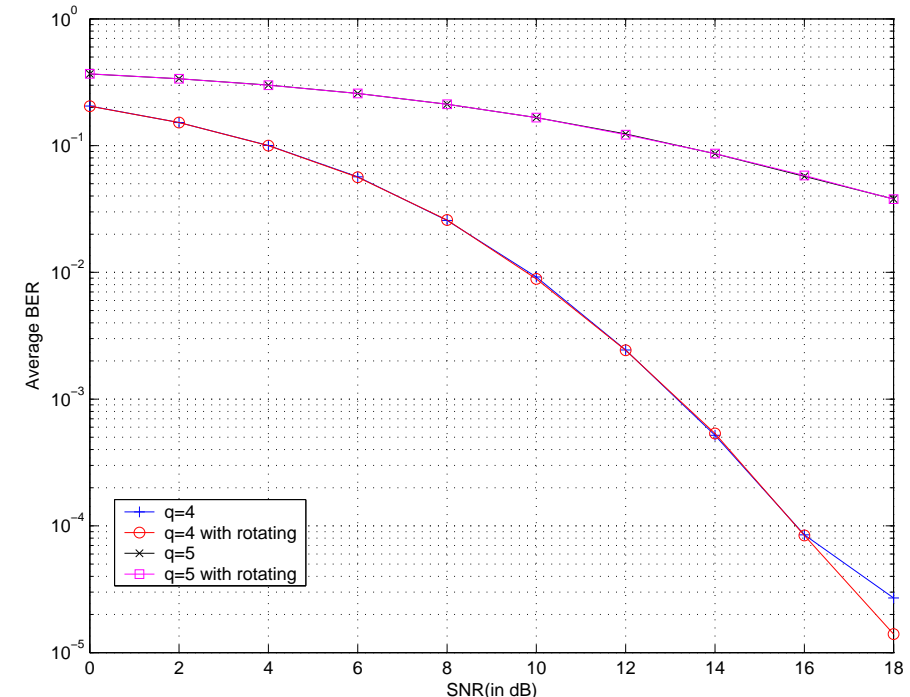

Fig. 3. Equal Error Precoder and Its Rotation Improvement

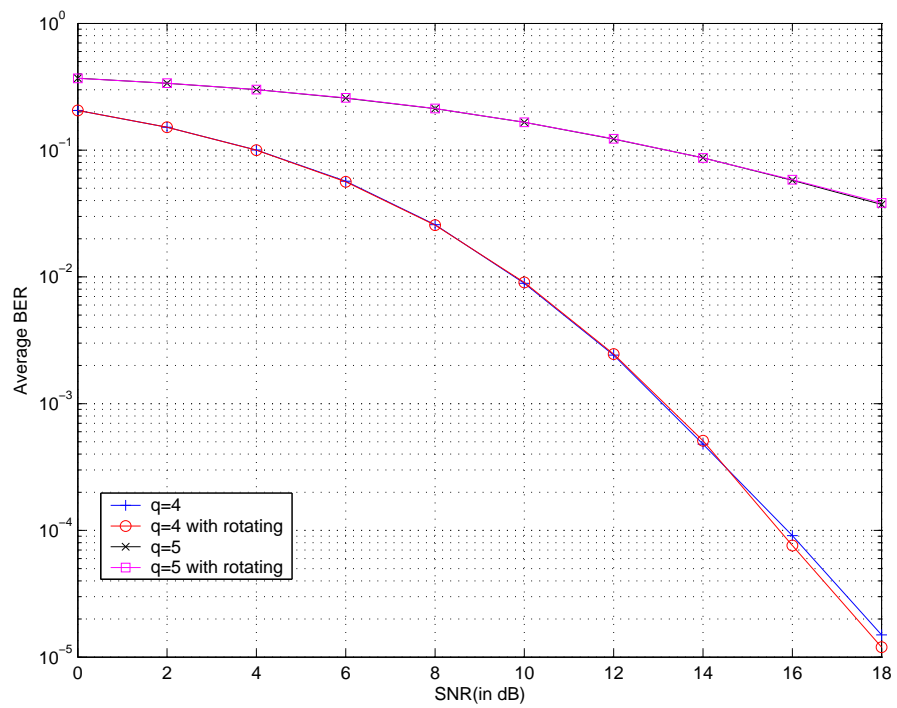

Fig. 4. Minimum PEP Precoder and Its Rotation Improvement

\section{B. Combinations with large improvement}

The un-weighted MMSE precoder and maximum information precoder have various subchannel SNR. Their performance improvements can be seen from Fig. 5 and 6 respectively. For $q=4$ of un-weighted MMSE, there is about $2.0 \mathrm{~dB}$ gain when $B E R=10^{-3}$ after rotating. From Fig. 6, system of maximum information design can obtain 3.0dB at $B E R=10^{-4}$ in the case of $q=4$. It seems that the systems can get more gain when SNR is higher. This can be explained by the asymptotic property of diversity and coding gains achieved by signal space diversity. Without the rotation, The performance of the un-weighted MMSE precoders is very close to the equal error one. However after rotation, the MMSE's BER outperforms the equal error design. This also demonstrates our conclusions in section IV.

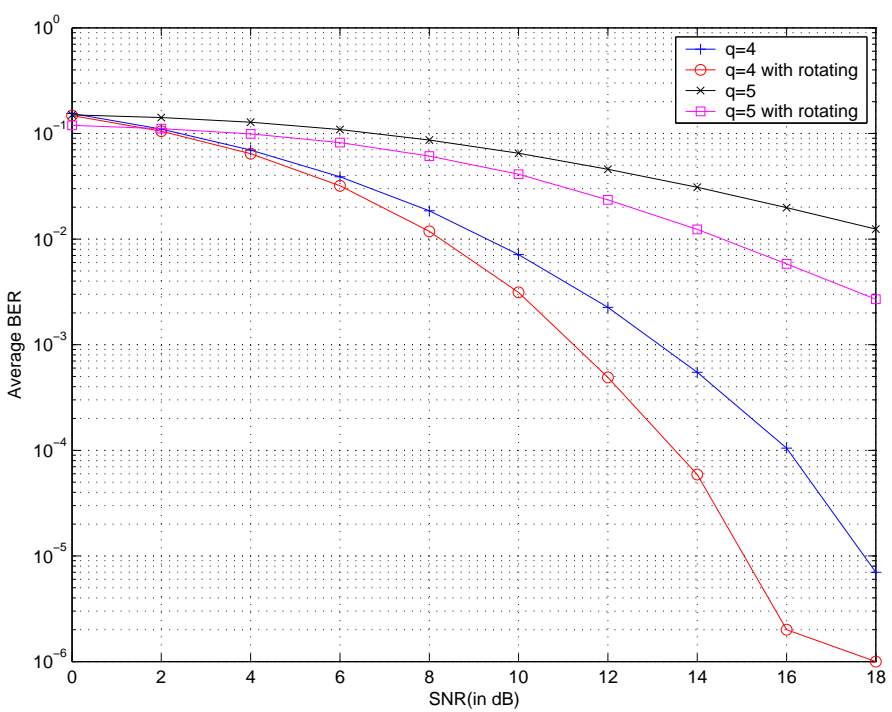

Fig. 5. Unweighted MMSE Precoder and Its Rotation Improvement

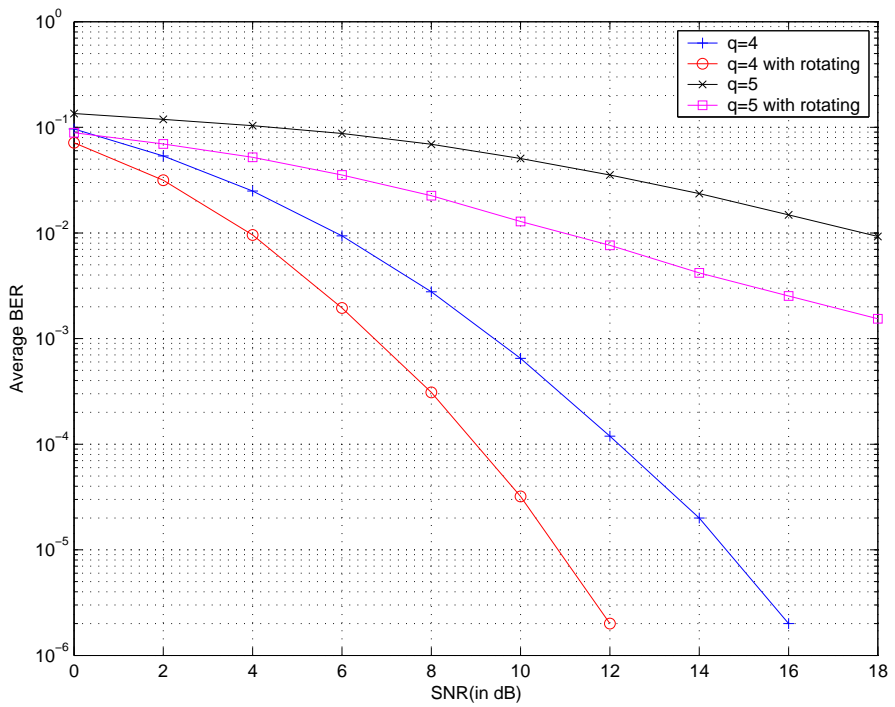

Fig. 6. Maximum Information Precoder and Its Rotation Improvement

\section{CONCLUSION}

In this paper, we have proposed a new scheme for adaptive MIMO system, which generalize the precoder design and the constellation rotation design to achieve better performance. The principle of our design has been discussed. The results can be used to determined how to design an enhanced adaptive MIMO system with no rate loss. Simulation results have backed up our results. To consider optimizing the two methods altogether must be a very interesting work.

\section{REFERENCES}

[1] P. Wolniansky, G. Foschini, G. Golden, and R. Valenzuela, "V-blast: an architecture for realizing very high data rates over the rich-scattering wireless channel," 1998. 
[2] J. Boutros and E. Viterbo, "Signal space diversity: A power- and bandwidth-efficient diversity technique for the rayleigh fading channel," IEEE Trans. Inform. Theory, vol. 44, July 1998.

[3] Y. Xin, Z. Wang, and G. B. Giannakis, "Space-time diversity systems based on linear constellation precoding," IEEE Trans. Wireless Communications, vol. 2, Mar. 2003.

[4] Y. Xin, "Space-time linear constellation precoded systems," Ph.D. dissertation, University Of Minnesota, 2003

[5] H. Sampath, P. Stoica, and A. Paulraj, "Generalized linear precoder and decoder design for mimo channels using the weighted mmse criterion," IEEE Trans. Commun., vol. 49, Dec. 2001.

[6] A. Scaglione, P. Stoica, and S. Barbarossa, "Optimal designs for spacetime linear precoders and decoders," IEEE Trans. Signal Processing, vol. 50, May 2002.

[7] H. Sampath, "Linear precoding and decoding for multiple input multiple output(mimo) wireless channels," Ph.D. dissertation, Stanford University, 2001. 